\title{
Stochastic optimization-based study of dimerization kinetics
}

\author{
SRIJEETA TALUKDER ${ }^{\mathrm{a}}$, SHRABANI SEN ${ }^{\mathrm{a}}$, RALF METZLER $^{\mathrm{b}, \mathrm{c}, *}$, \\ SUMAN K BANIK $^{\mathrm{d}, *}$ and PINAKI CHAUDHURY ${ }^{\mathrm{a}, *}$ \\ aDepartment of Chemistry, University of Calcutta, 92 A P C Road, Kolkata 700 009, India \\ ${ }^{\mathrm{d}}$ Institute for Physics and Astronomy, University of Potsdam, D-14476 Potsdam-Golm, Germany

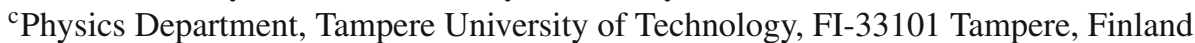 \\ ${ }^{\mathrm{d}}$ Department of Chemistry, Bose Institute, 93/1 A P C Road, Kolkata 700 009, India \\ e-mail: rmetzler@uni-potsdam.de; skbanik@bic.boseinst.ernet.in; pinakc@ rediffmail.com
}

MS received 8 March 2013; revised 2 July 2013; accepted 12 August 2013

\begin{abstract}
We investigate the potential of numerical algorithms to decipher the kinetic parameters involved in multi-step chemical reactions. To this end, we study dimerization kinetics of protein as a model system. We follow the dimerization kinetics using a stochastic simulation algorithm and combine it with three different optimization techniques (genetic algorithm, simulated annealing and parallel tempering) to obtain the rate constants involved in each reaction step. We find good convergence of the numerical scheme to the rate constants of the process. We also perform a sensitivity test on the reaction kinetic parameters to see the relative effects of the parameters for the associated profile of the monomer/dimer distribution.
\end{abstract}

Keywords. Stochastic optimization; dimerization kinetics; sensitivity analysis; stochastic simulation algorithm; probability distribution function.

\section{Introduction}

The principles of chemical kinetics constitute one of the cornerstones in the study of chemical and biological reaction networks. Evaluation of correct individual step-based pathways (both the nature of the reaction as well as the correct magnitude of the rate constant) present in a multi-step reaction scheme is central in establishing a complete reaction model in any multistep reaction process. The conventional way to study reaction kinetics is to write down the mean field rate equations for the process, integrate them and follow the variation in the concentration of each species involved as a function of time. However, this strategy is too simplistic and fails in situations where the number of reacting species is small, since for small number of particles, fluctuations in the species population become relevant. In the conventional rate equations approach, it is assumed that the process is both continuous and deterministic. However, in reality, in particular in biochemical reactions in living cells, these assumptions often fail. Thus, reactants may occur at nanomolar rates. Reaction kinetics at such low concentrations are intrinsically discrete and stochastic. The stochastic

*For correspondence simulation algorithm (SSA) is an elegant formulation to incorporate these effects and predict correct results in a complicated multi-step reaction network. ${ }^{1,2}$ Applications of SSA range from the study of simple schemes such as two-step consecutive reactions or parallel reactions, ${ }^{2}$ to biological systems such as the dynamics of biopolymers such as DNA. ${ }^{3,4}$ The rate constants associated with each individual step in a multi-step reaction scheme might not always be known a priori, or there might be a range of values of the rate constants, for which predictions for the overall reaction are compatible with experimental data. The correct prediction of all individual rate constants is not always an easy task and involves an optimization process. If an optimization scheme can be linked to SSA, then it should be possible to evaluate a correct set of reaction parameters, quantifying the complete kinetic behaviour of a reaction network. Here, we analyse in detail the application of stochastic optimization schemes to the dimerization kinetics of proteins. It is also an experimentally well-studied kinetics. ${ }^{5,6}$

Optimization schemes can generally be classified into two categories, deterministic and stochastic optimization. The main difference between these two schemes is that deterministic ones are not truly global optimizers, whereas the stochastic ones are. Stochastic optimizers are not gradient-based and incorporate the 
principle of stochasticity to arrive at correct solutions. Here, we use three techniques separately in conjunction with the SSA scheme to evaluate the correct set of reaction rate constants, these being simulated annealing (SA), genetic algorithm (GA) and parallel tempering (PT). These algorithms are also known as natural algorithms as they draw their working philosophy from natural processes. In particular, they are robust and able to find solutions to complex problems with consummate ease, unlike deterministic methods. Let us summarize the fundamental properties of the three stochastic optimizers:

(i) SA is a global optimization technique which mimics the process of annealing in metallurgy to design a mathematical optimization scheme. Thus, the energy landscape of the search space is initially sampled at a high temperature, such that thermal fluctuations may easily lift the optimizer out of deeper minima. On decreasing the temperature, the search is guided towards the global minimum. SA has been widely used in the last few decades with widespread applications ranging from the solution of the travelling salesman problem, solving differential equations, finding structures of novel materials and studies involving structure and dynamics in quantum chemistry. ${ }^{7-15}$

(ii) PT is a method based on replica exchange among randomly created configurations. PT has been successfully used to solve highly dimensional optimization problems with applications in the determination of structural features in proteins and other polymeric materials, the study of spin glasses and other solid state systems, or phase transitions in clusters formed by hydrogen bonding or other van-der Waals forces. ${ }^{16-23}$

(iii) GA uses the concepts of genetics and mimics the natural process of selection, crossover and mutation as present in living systems. GA has been widely used, its applications ranging from solutions of differential equations, geometry optimization in large molecules and clusters, or the design of laser pulses to follow dissociation dynamics of molecular systems. ${ }^{24-33}$

As we are interested in the evaluation of the correct kinetic rate parameters of a multi-step reaction, it is important to know a priori the relative importance of each parameter. Sensitivity analysis is an important statistical technique which can give us an insight into this question. Such sensitivity analyses have been used widely to assess how individual parameters influence the dynamics when perturbed from their expected values. ${ }^{34,35}$ Such an analysis provides relevant information to decide the actual reaction scheme of some chemical kinetics. If a parameter is more sensitive than the other, small deviations from the mean value will leave its mark on the kinetics by introducing large deviations from expected trends, while for insensitive parameters, the effect will be minimal. One can devise various measures for quantitatively calculating the sensitivity measures for various parameters such as the Fourier amplitude based sensitivity test, ${ }^{36,37}$ and others. This analysis leads to a more in-depth understanding of any kinetic scheme.

Here, we follow the three combined schemes: $\mathrm{GA}+\mathrm{SSA}, \mathrm{SA}+\mathrm{SSA}$ and PT $+\mathrm{SSA}$ to evaluate the correct set of rate constants necessary to explain all features in the reaction scheme of the dimerization of a protein. We perform a sensitivity test on the rate parameters in our model and use the findings to see if an optimization strategy influenced by the sensitivity test can lead to quicker convergence.

\section{Dimerization model}

In our study, we have chosen a minimal kinetic scheme of protein dimerization, ${ }^{38}$ for which the minimal kinetic steps can be described by the following elementary reactions:

$$
\begin{gathered}
\varnothing \stackrel{k_{1}}{\longrightarrow} M, \\
M \stackrel{k_{2}}{\longrightarrow} \emptyset, \\
M+M \stackrel{k_{3}}{\longrightarrow} D, \\
D \stackrel{k_{4}}{\longrightarrow} M+M, \\
D \stackrel{k_{5}}{\longrightarrow} \emptyset,
\end{gathered}
$$

where the $k_{i}$ are the rate constants for the individual reactions steps (1a) to (1e), and $M$ and $D$ denote the number of monomers and dimers of the protein, respectively. To keep the dynamics simple, we neglect effects of cell growth and cell division in the model, i.e., we assume that the reaction occurs at fixed volume.

The marginal probability distribution function for monomer and dimer can be defined as

$$
\begin{aligned}
& \widetilde{P}_{x}(y, t)=\int P(x, y, t) d x, \\
& \widetilde{P}_{y}(x, t)=\int p(x, y, t) d y,
\end{aligned}
$$

where $x$ and $y$ are the number of monomer and dimer molecules at time $t$, respectively. As the system deals with small numbers of molecules, the time evaluation 
should be obtained by using stochastic formulation, i.e., by solving master equation. However, it is often quite tedious to solve such a master equation analytically for complicated systems similar to the present one. Consequently, one has to resort to numerical methods to quantify the underlying process, for instance by using SSA.

Typically, in SSA, a probability density function $P(\tau, \mu) d \tau$ is introduced for a given state at time $t$, which is a measure for the probability that within the infinitesimal time interval $(t+\tau, t+\tau+d \tau)$, the $\mu$ th reaction will occur, where the index $\mu$ stands for a given reaction step. $P(\tau, \mu) d \tau$ is supposed to follow Poissonian statistics such that

$$
P(\tau, \mu)=\alpha_{\mu} \exp -\left(\alpha_{0} \tau\right),
$$

where

$$
\alpha_{\mu}=h_{\mu} c_{\mu}, \alpha_{0}=\sum_{\mu=1}^{N} h_{\mu} c_{\mu}
$$

In the latter relation, $h_{\mu}$ is the number of molecules of the species involved in the $\mu$ th reaction step, $c_{\mu}$ is the respective rate constant and $N$ is the number of reaction channel. The time step $\tau$ is defined via

$$
\tau=\frac{1}{a_{0}} \ln \frac{1}{r_{1}} .
$$

In SSA, the underlying randomness is introduced in the choice of $\tau$ and $\mu: \mu$ be the integer for which

$$
\sum_{v=1}^{\mu-1} a_{v}<r_{2} a_{0} \leq \sum_{v=1}^{\mu} a_{v},
$$

where $r_{1}$ and $r_{2}$ are random numbers between 0 to $1 .^{1,2}$ $\tau$, in eq. (5), thus can never be negative as $r_{1} \leq 1$ (the derivation of eq. (5) is given in Appendix). Utilizing SSA in the present reaction scheme given by eqs (1a-e), one may calculate the equilibrium marginal distribution profile separately for monomer and dimer.

\section{Stochastic optimization}

We employ three optimization techniques SA, GA, and PT to determine the optimum set of kinetic parameters in our kinetic scheme. These optimization techniques are not gradient-based and use stochastic principles, hence are known as stochastic optimizers. All the simulations are started with an arbitrary parameter set, which is obtained by perturbing the literature value of the rate constants in the dimerization of protein to a preset extent. The same initial parameter set has been taken for all the three optimization schemes. To follow the progress of the optimization, we compare the probability distribution profile of the monomers and dimers of the protein obtained by using the literature value of the rate constants in the SSA with the distribution profile for various sets of rate constants obtained from different iterations of the simulations. The parameters are said to be optimized if the distribution profile for a set of parameters coincides with the distribution profile from literature within $0.01 \%$.

During the simulation, the parameter set of rate constants obtained in each iteration is fed into the SSA to produce the distribution profile corresponding to this output of the optimizer. Then, an objective function, popularly known as the cost function, is calculated to measure the extent of difference between the present distribution profile and the profile obtained for the literature value of the parameter set (expected distribution profile). The cost function is basically the cumulative differences in probabilities for the two distribution profiles, for different species (in our system for the monomer and the dimer only). While calculating the difference in the two distributions, magnitudes of the two functions at certain discrete points have been taken. The index ' $i$ ' in eq. (7) refers to the discrete points, at which the values of the two functions have been compared.

$$
\text { cost }=\sum_{m=1}^{n} \sum_{i=1}^{k}\left(\widetilde{P}_{l}\left(m_{i}\right)-\widetilde{P}\left(m_{i}\right)\right)^{2}
$$

where $\widetilde{P}_{l}\left(m_{i}\right)$ and $\widetilde{P}\left(m_{i}\right)$ are the probabilities of the $m$ th species at the $i$ th grid point (whereas, $n$ is the total number of species and $k$ is the total number of grid points) in the distribution profile obtained by feeding the literature value of the rate constants ${ }^{38}$ in SSA and the profile for the rate parameters at a optimization step respectively. The cost has to be minimized with simulation and for the optimum solution it must tend to zero. Figure 1 shows the distribution profiles for literature value and for a set of optimized parameters, which actually coincides with the objective distribution profile.

Assigning of cost function utterly depends on the specificity of the problem. The above equation may not be the ultimate way to define cost function. One may define cost with respect to the time series profile of mean and variance also. Then, it becomes our objective to reach the mean and variance profile for the literature value, in course of optimization.

$$
\begin{aligned}
\operatorname{cost}= & \sum_{m=1}^{n} \sum_{i}^{k_{1}}\left([m]_{i}^{l}-[m]_{i}\right)^{2} \\
& +\left(\operatorname{Var}(m)_{i}^{1}-\operatorname{Var}(m)_{i}\right)^{2},
\end{aligned}
$$




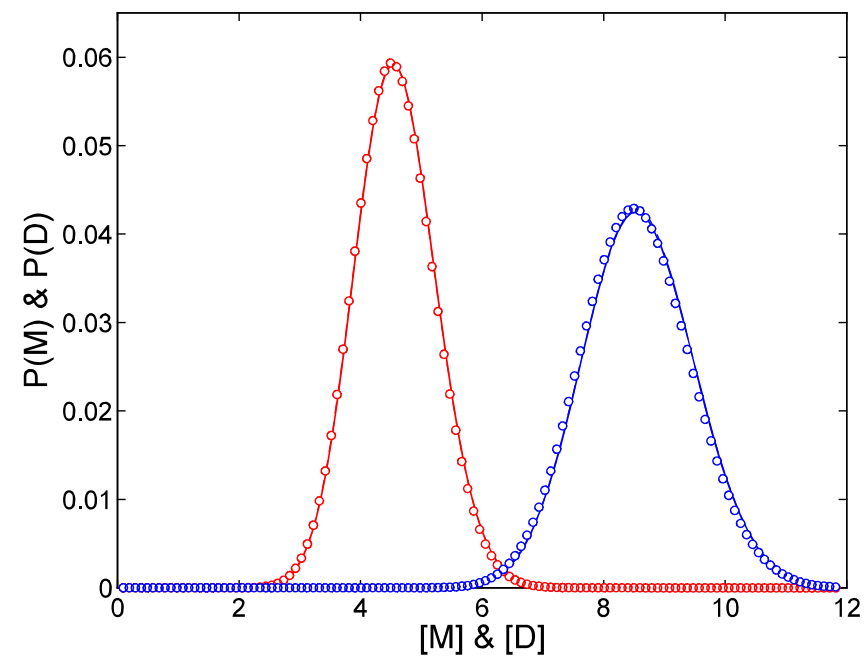

Figure 1. Plot of distribution profile of monomer and dimer. Solid line denotes the profile for literature value of rate parameters and the open circles represent the profile for optimized set. Red and blue colours depict monomer and dimer, respectively.

$[m]_{i}$ and $\operatorname{Var}(m)_{i}$ are the concentration and the variance of $m$ th species at $i$ th time, respectively. Term with notation $l$ is for the expected profile and the other is obtained from simulation. In this case also, the cost would be theoretically zero for the optimal solution. Figure 2 represents such profile for literature value of rate constants and the profile for the set of rate constant acquired from an optimization run.
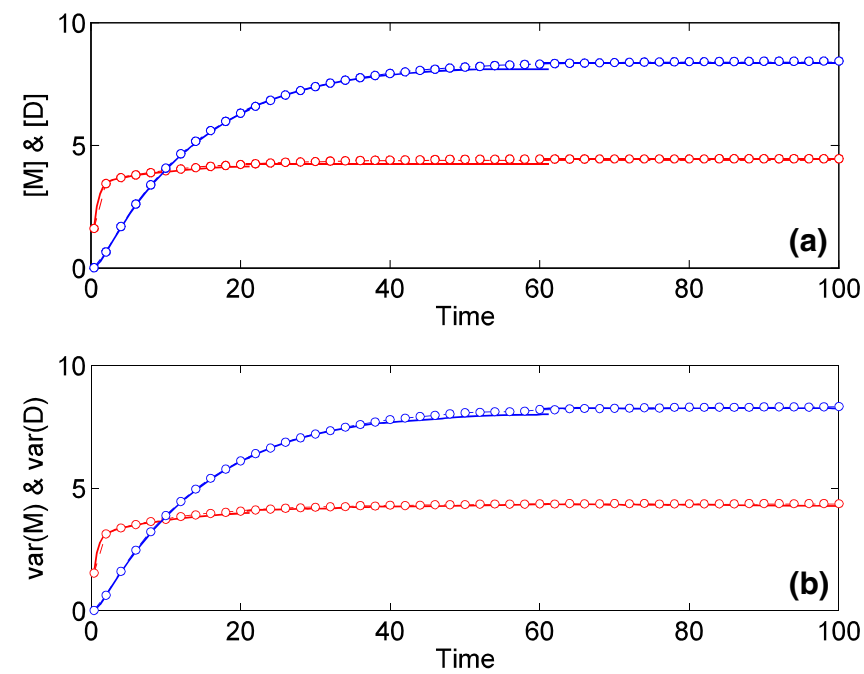

Figure 2. Plot of mean and variance of monomer and dimer concentration with time. Panel (a) is the time series of mean and panel (b) is that of variance. Solid line denotes the profile for literature value of rate parameters and open circles represent the profile for optimized set. Red and blue colours depict monomer and dimer, respectively.

\section{Sensitivity analysis}

Generally, in any chemical or biochemical network, not all the parameters hold equal priority. A sensitivity analysis is conducted to determine which input parameters contribute the most to the output variable, which parameters are insignificant, whether the input parameters do interact among themselves, whether the interaction is physically explainable, and, after all, to search for the optimal regions within the parameters space for use in a subsequent calibration study. One can say, a system is sensitive with respect to a parameter if a small change to this parameter affects the output abruptly.

For a quantitative estimation of the sensitivity of the rate parameters, we use a variance-based sensitivity analysis test. The idea of this analysis is taken from Saltelli et $a{ }^{34}{ }^{34}$ They report a comparative discussion of different sensitivity analysis techniques in order to reduce the computational cost of running the model. The main idea was developed by Cukier and coworkers $^{36,37}$ in the 1970, and was known as Fourier amplitude sensitivity test (FAST). In the present study, we adopt the implementation of the FAST-based sensitivity test as used by Saltelli et al. ${ }^{34}$

The variance in output with input parameter set having one parameter fixed at some value is defined by the term $V_{X_{-i}}\left(Y \mid X_{i}=X_{i}^{*}\right)$, where $Y$ is the output factor and the subscript $X_{-i}$ of $V$ denotes that the variance is taken over all other input parameters other than $X_{i}$ which is fixed at $X_{i}^{*}$. This is generally less than the variance with fully random input set $V(Y)$, but may depend on the magnitude of the fixed parameter. To remove this type of parameter dependence, an average of the variance over the different values of the fixed parameter is estimated by $E_{X_{i}}\left(V_{X_{-i}}\left(Y \mid X_{i}=X_{i}^{*}\right)\right)$. We may write the total variance $V(Y)$ as follows: ${ }^{34}$

$$
V(Y)=E_{X_{i}}\left(V_{X_{-i}}\left(Y \mid X_{i}\right)\right)+V_{X_{i}}\left(E_{X_{-i}}\left(Y \mid X_{i}\right)\right) .
$$

Thus a FAST-based sensitivity index is defined simply as

$$
S_{i}=\frac{V_{X_{i}}\left(E_{X_{-i}}\left(Y \mid X_{i}\right)\right)}{V(Y)} .
$$

Using the above-mentioned idea, we discuss the sensitivity of the model parameters in the next section.

\section{Results and discussion}

Our main focus is to evaluate the optimal set of kinetic parameters for the scheme given by eqs (1a-1e) of protein dimerization. All stochastic optimizers: SA, GA, 
Table 1. Comparison of kinetic parameter values. Units of $k_{1}, k_{2}, k_{3}, k_{4}$ and $k_{5}$ are $\mathrm{nM} \min ^{-1}, \min ^{-1}, \mathrm{nM}^{-1} \min ^{-1}$, $\min ^{-1}$ and $\min ^{-1}$, respectively.

\begin{tabular}{lcccc}
\hline & & \multicolumn{3}{c}{ Simulation } \\
\cline { 3 - 5 } Parameter & Literature $^{38}$ & SA & GA & PT \\
\hline$k_{1}$ & 50.0 & 49.42 & 49.41 & 49.44 \\
$k_{2}$ & 1.02 & 1.007 & 1.008 & 1.009 \\
$k_{3}$ & 0.01 & 0.009 & 0.009 & 0.009 \\
$k_{4}$ & 0.1 & 0.10 & 0.099 & 0.098 \\
$k_{5}$ & 0.2 & 0.019 & 0.019 & 0.019 \\
\hline
\end{tabular}

and PT, turned out to decipher the optimal set of kinetic parameters. Rate constants obtained from simulations are in good proximity to the literature value. The optimized parameter values (average of the five runs) in each simulation procedure, as well as the literature values ${ }^{38}$ are shown in table 1 .

We followed the approach to the converged results for the five kinetic parameters of the protein dimerization model in three different schemes. The results are shown in figure 3. For each technique, we show five simulation runs, as shown in the graph. The literature value of each parameter is shown as black dashed line.
The parameters $k_{1}$ and $k_{2}$ show a good convergence within a very short range around the literature value, but the other rate parameters show a spread (for the reason, see the discussion below). It is also evident from figure 3 that GA and PT runs take fewer steps (about 60 to 70) to converge to reasonably convincing solutions, while SA takes about 100 steps. However, in terms of computational time required, GA seems to be the most efficient method followed by SA and PT. This is expected on theoretical lines, as GA and PT process a number of trial solutions simultaneously, while SA improves on a single starting solution.

Let us now study sensitivity analysis to classify the most delicate rate constants. We apply a fixed perturbation $(5 \%)$ onto a given rate constants at a time, keeping the other rate parameters unperturbed with respect to the literature value. We then use SSA to return the equilibrium probability distribution for the perturbed run. By comparing the discussed distribution function with the theoretical one, we qualitatively judge the sensitivity of the rate parameters (plots shown in figure 4). In our case, the dimerization kinetics is more sensitive to $k_{1}$ and $k_{2}$ than the others. This result justifies the observation in figure 3 . The more sensitive parameters should converge to a small range around the target value, whereas relatively less sensitive ones exhibit a wider spread at the end of optimization.
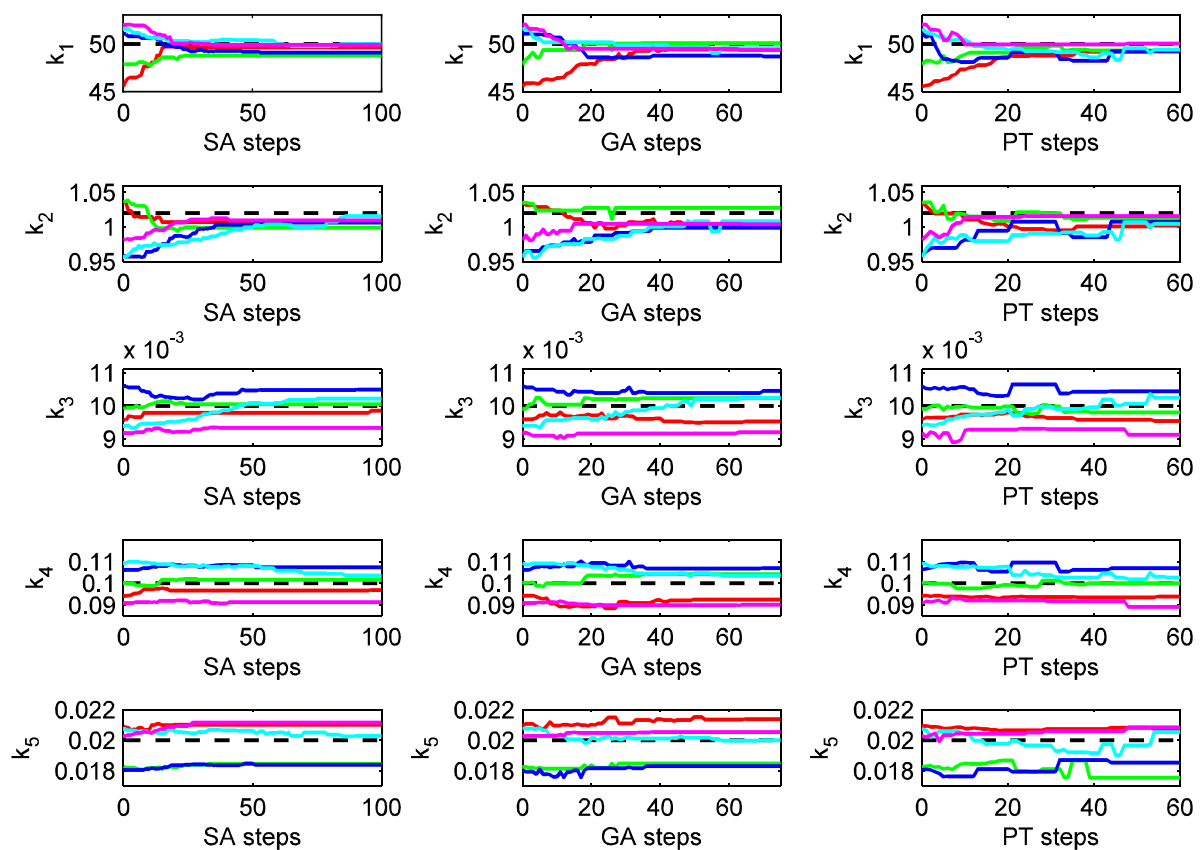

Figure 3. (colour online) Kinetic parameters $\left(k_{1}, k_{2}, k_{3}, k_{4}\right.$, and $\left.k_{5}\right)$ versus number of SA, GA and PT steps. Black dashed line represents values of kinetic parameters taken from Adalsteinsson et al., ${ }^{38}$ while the step-like lines (red, blue, green, cyan and magenta) are the results of five different SA, GA, and PT runs. 

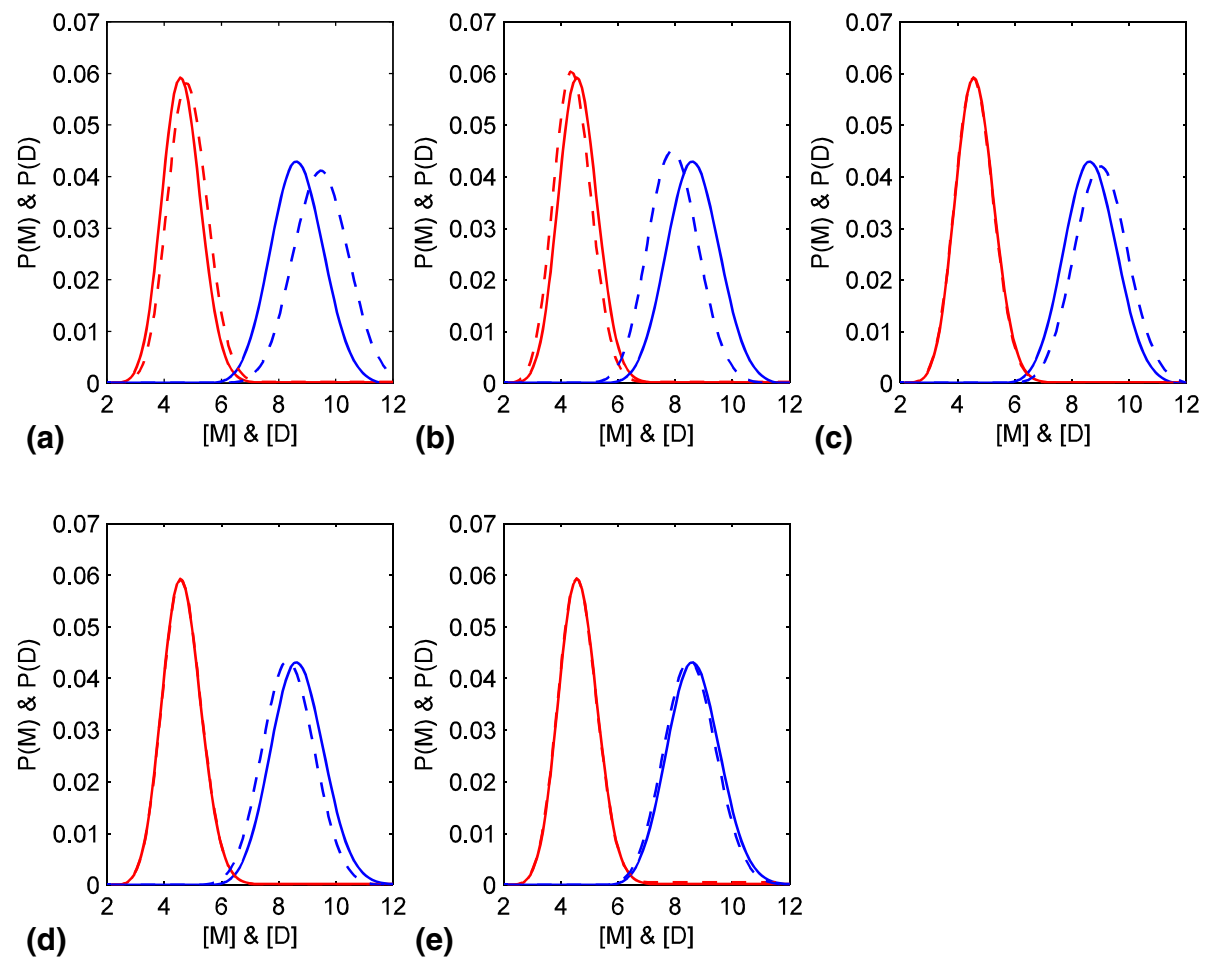

Figure 4. (colour online) Expected probability distribution (solid line) and distribution obtained from SSA by perturbing one parameter at a time (dashed lines). Red and blue represent monomer and dimer distributions, respectively. In panels (a) to (e), the perturbed parameters were respectively, $k_{1}, k_{2}, k_{3}, k_{4}$ and $k_{5}$.

Sensitivity index $\left(S_{k_{i}}\right)$ for the output monomer and dimer concentrations are calculated separately with respect to each input parameter at different time for the protein dimerization kinetics. Figure 5 depicts the plot of sensitivity index against time. Higher sensitivity for a particular parameter indicates that the system becomes
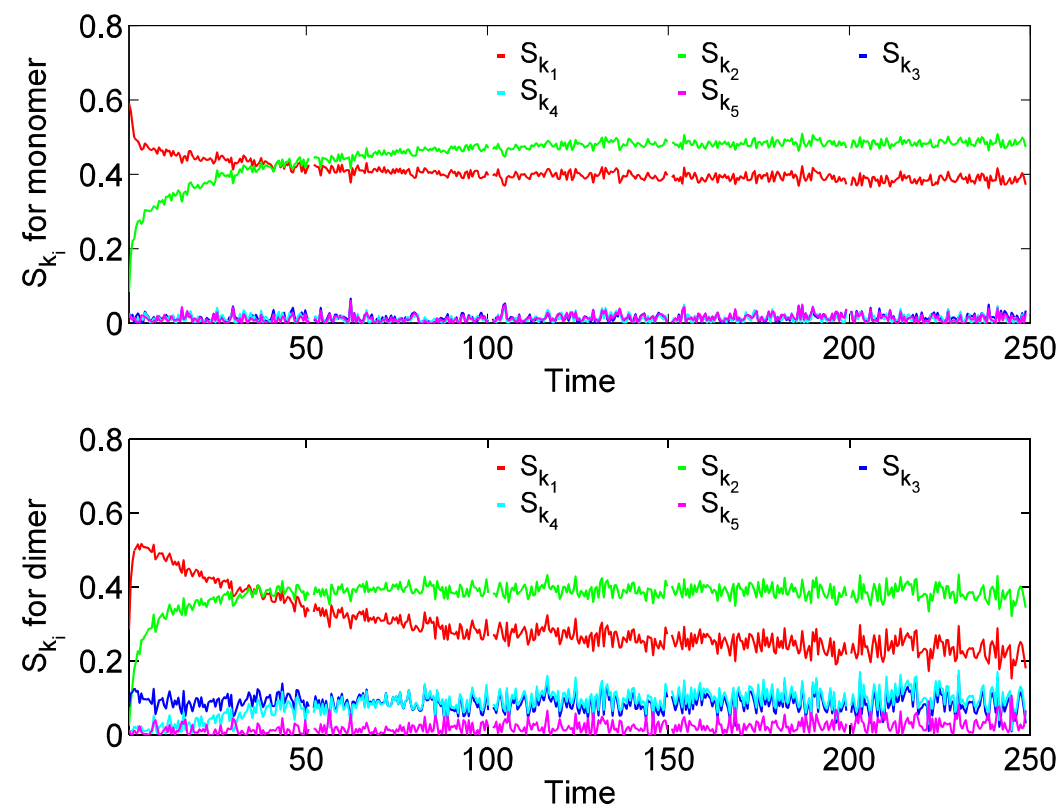

Figure 5. (colour online) Sensitivity index, Ski for $i=1-5$ versus time for monomers and dimers. The red, green, blue, magenta and cyan coloured lines depict $k_{1}, k_{2}, k_{3}, k_{4}, k_{5}$, respectively. 

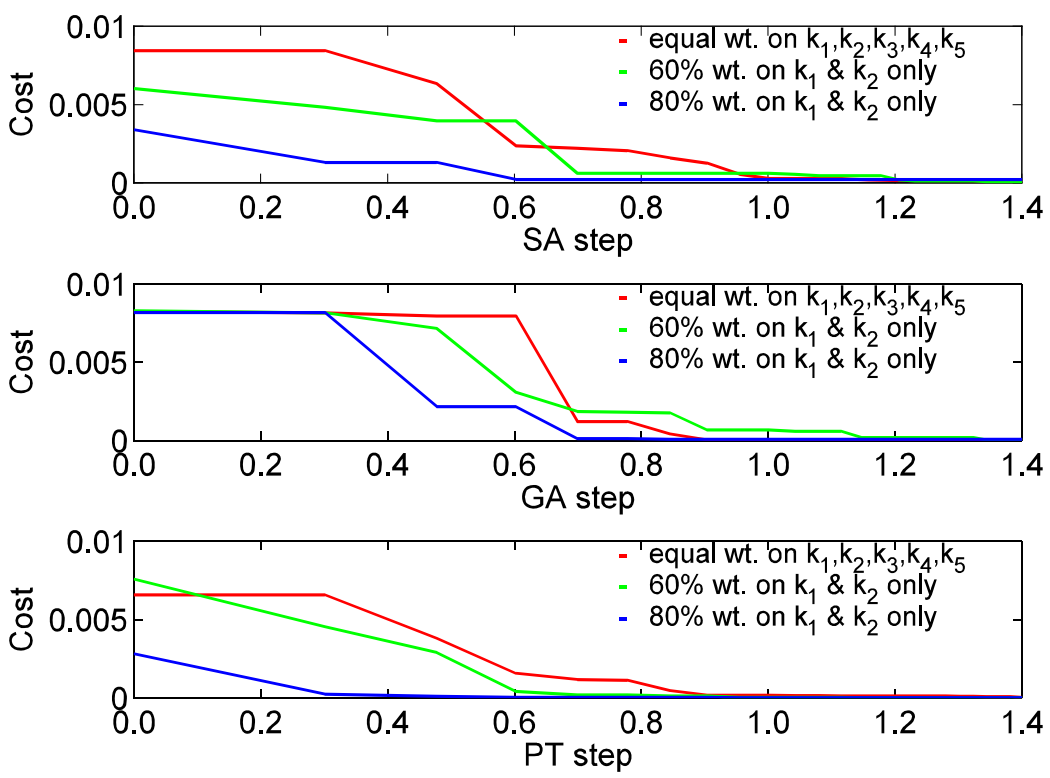

Figure 6. (colour online) Optimization profile against SA, GA, and PT steps (in log scale). Red line: Simulation with equal weight to all the kinetic parameters. Green line: Biased simulation by $60 \%$ weight on $k_{1}$ and $k_{2}$, Blue line: biased simulation by $80 \%$ weight on $k_{1}$ and $k_{2}$.

more sensitive to that parameter than the others. Our results depict that both the monomer and dimer concentrations are more sensitive with respect to $k_{1}$ and $k_{2}$ than to $k_{3}, k_{4}$, and $k_{5}$. Figure 5 also shows that as a function of time, $S_{k_{1}}$ decreases and $S_{k_{2}}$ increases, and a crossover of sensitivity occurs. This crossover reflects the physical idea that initially the system becomes sensitive to the rate constant, which produces monomers from a source. As time progresses, due to adequacy of monomer concentrations, sensitivity index shifts to the reverse rate constant of the reaction. This type of crossover is also observed on a smaller scale in $k_{3}$ and $k_{4}$.

Since $k_{1}$ and $k_{2}$ are reflected to be the more sensitive parameters, they have to be explored more than the rest of the rate parameters. This idea is incorporated during the parameter evolution by using stochastic optimization techniques. If we assign higher probability to the sensitive parameters, to be sampled than the others, convergence occurs rapidly during optimization. Figure 6 clearly shows that on applying $80 \%$ weight on $k_{1}$ and $k_{2}$ to be sampled (20\% weight on $k_{3}, k_{4}$ and $k_{5}$ ), the cost function falls more rapidly than the sampling with $60 \%$ weight on $k_{1}$ and $k_{2}\left(40 \%\right.$ weight on $k_{3}, k_{4}$ and $k_{5}$ ). This in turn is obviously faster than a run, in which equal weight is assigned for sampling of each rate parameter. The trends are in a similar line for runs involving SA, GA and PT. This strategy of doing biased optimization runs, involving higher weight to sample more sensitive parameters, will certainly contribute to the decrease in computational cost.
It is also important to conclude this section with an idea of the robustness of the three used numerical algorithms in deciphering the rate constants. In the present study, we have selected the initial trail set of rate constants from a Gaussian distribution, whose peak corresponds to the literature value and a half width of $10 \%$ of the respective rate constant. This is a moderately large perturbation. Convergence from this initial set is quite close to the values reported in literature. As we have also done a sensitivity analysis on each of the five calculated reaction rate parameters, a check can also be made on the relative importance/rigidity that an evaluated value can have. Lesser the sensitivity of a particular data, greater the spread of the reported value, even while matching the correct dimer-monomer distribution profile.

\section{Conclusion}

We have shown that stochastic optimization techniques in conjunction with SSA can help determining kinetic parameters in multi-step kinetic schemes. All the three optimizers (SA, GA and PT) perform equally well to predict the values of the rate constants. We have also shown that an optimization study guided by findings from sensitivity analysis can help us distinguish between the parameters based on its importance and if these are incorporated into the optimization, a quicker convergence can be achieved. This strategy of initially 
doing a sensitivity analysis and segregating the rate parameters into zones of importance and then subsequently using stochastic optimization techniques to decipher them can be an important strategy for studying kinetics in complex biochemical networks, where rate parameters can be numerous. The number of ordinary differential equations to be solved in such cases are also very large. An unbiased strategy of allocating equal samplings to each and every rate parameter will make the process of finding the solution, computationally costly and tedious. A stochastic search in conjunction with the sensitivity analysis will be much more efficient.

\section{Acknowledgements}

ST acknowledges the financial support from the University Grants Commission (UGC), New Delhi, for granting a Senior Research Fellowship. SS thanks the UGC, New Delhi for granting a DS Kothari Post-Doctoral Fellowship. RM acknowledges funding through the Academy of Finland's FiDiPro scheme. PC wishes to thank the Centre for Research on Nano Science and Nano Technology, University of Calcutta for a research grant [Conv/002/Nano RAC (2008)]. SKB acknowledges support from Bose Institute through Institutional Programme VI - Development of Systems Biology.

\section{Appendix A}

To generate a real random number $x$, which follows a probability density function $P(x)$, one has to consider another function $F(x)$.

$$
F(x)=\int_{-\infty}^{x} P\left(x^{\prime}\right) d x^{\prime} .
$$

$F(x)$ is the probability distribution function which satisfy

$$
F(x)=r,
$$

where $r$ is basically a random number from the uniform distribution between 0 and 1 .

Then,

$$
x=F^{-1}(r) .
$$

In eq. (5) $\tau$ (a real random number), follows the probability density function $P(\tau) ., 1,2$

$$
\begin{array}{ll}
P(\tau)=a_{0} \exp \left(-a_{0} \tau\right) & \text { for } 0 \leq \tau \leq \infty \\
P(\tau)=0 & \text { elsewhere. }
\end{array}
$$

Now,

$$
\begin{aligned}
& F(\tau)=\int_{0}^{\tau} P\left(\tau^{\prime}\right) d \tau^{\prime} \\
& r=1-\exp \left(-a_{0} \tau\right) .
\end{aligned}
$$

$(1-r)$ is also a random number, thus $\tau$ becomes

$$
\tau=\frac{1}{a_{0}} \operatorname{In} \frac{1}{r} .
$$

\section{References}

1. Gillespie D T 1976 J. Comp. Phys. 22403

2. Gillespie D T 1977 J. Phys. Chem. 812340

3. Chaudhury P, Metzler R and Banik S K 2009 J. Phys. A42 335101

4. Talukder S, Chaudhury P, Metzler R and Banik S K 2011 J. Chem. Phys. 135165103

5. Jia H, Satumba W J, Bidwell III G L and Mossing M C 2005 J. Mol. Biol. 350919

6. Venezia C F, Meany B J, Braz V A and Barkley M D 2009 Biochemistry 489084

7. Kirkpatrick K S, Gelatt C D and Vecchi M P 1983 Science 220671

8. Wilson S R and Cui W L 1990 Biopolymers 29225

9. Andricioaei I and Straub J E 1996 Phys. Rev. E53 3055

10. Zhang J 2011 J. Mol. Model. 17173

11. Van Setter M J, Lohstroh W and Fichtner M 2009 J. Mater. Chem. 197081

12. Field M J 1995 J. Chem. Phys. 1033621

13. Dutta P, Mazumdar D and Bhattacharyya S P 1991 Chem. Phys. Lett. 181293

14. Biring S K and Chaudhury P 2010 Chem. Phys. 37746

15. Guha S, Roy S and Chaudhury P 2011 Struct. Chem. 22 1007

16. Earl D J and Deem M W 2005 Phys. Chem. Chem. Phys. 73910

17. Yan Q and de Pablo J J 1999 J. Chem. Phys. 1119509

18. Li Y, Protopopescu V A, Arnold N, Zhang X and Goris A 2009 Appl. Math. Comput. 212216

19. Fiore C E 2008 Phys. Rev. E78 041109

20. Patriksson A and van der Spoel D 2008 Phys. Chem. Chem. Phys. 102073

21. Yan S and de Pablo J J 2000 J. Chem. Phys. 1131276

22. Tsai H H, Reches M, Tsai C J, Gunasekaran K, Gazit E and Nussinov R 2005 Proc. Natl. Acad. Sci. USA 102 8174

23. Falcioni M and Deem M W 1999 J. Chem. Phys. 110 1754

24. Goldberg D E 1989 Genetic algorithm in search, optimization and machine learning (Reading, MA: Addison Wesley)

25. Zeiri Y, Fattal E and Koslof R 1995 J. Chem. Phys. 102 1859

26. Saha R, Chaudhury P and Bhattacharyya S P 2001 Phys. Lett. A291 397

27. Hennessy M H and Kelly A M 2004 Phys. Chem. Chem. Phys. 61085

28. Zacharias C R, Lemes M R and Dal Pino Jr A 1998 J. Mol. Struct. (Theochem) $\mathbf{4 3 0} 29$ 
29. Johnston R L 2003 Dalton Trans. 4193

30. Weigend F and Ahlrichs A 2010 Philos. Trans. R. Soc. A368 1245

31. Sharma S, Singh H and Balint-kurti G G 2010 J. Chem. Phys. 132064108

32. Musin R R and Zheltikov A M 2008 Optics Commun. 281567

33. Riaz S S, Sharma R, Bhattacharyya S P and Roy D S 2007 J. Chem. Phys. 127064503
34. Salteli A, Ratto M, Tarantola S and Campolongo F 2005 Chem. Rev. 1052811

35. Turanyi T 1990 J. Math. Chem. 5203

36. Cuckier R I, Fortuin C M, Schuler K E, Petschek A G and Schailby J H 1973 J. Chem. Phys. 593873

37. Cuckier R I, Schailby J H, and Schuler K E 1973 J. Chem. Phys. 631140

38. Adalsteinsson D, McMillen D and Elston T C 2005 BMC Bioinformatics 524 